\title{
Cardiovascular disease in patients with type 2 diabetes mellitus: vulnerable plaques and vulnerable blood
}

\author{
GN Viswanathan and AG Zaman
}

\section{Introduction}

The prevalence of diabetes mellitus is rising at an exponential rate globally, with type 2 diabetes mellitus (T2DM) the most common form. The presence of coronary artery disease in patients with T2DM results in significant mortality and morbidity. The relative risk of cardiovascular events in those with T2DM remains unchanged despite good management of the diabetes mellitus and other cardiovascular risk factors. Recurrent cardiovascular thrombotic events are more common in coronary artery disease patients who have T2DM than in those without T2DM, despite current 'gold standard' secondary prevention therapy. ${ }^{1}$ Blood thrombogenicity (the ability of blood to clot or 'blood vulnerability') is greater in T2DM patients and is associated with poorer outcomes. Better understanding and identification of novel therapeutic targets that can be used to reduce thrombogenicity might improve clinical outcomes in this high-risk population.

\section{Epidemiology of diabetes mellitus}

It is estimated that there will be 4 million people in the UK who have diabetes by 2025, and the majority will have T2DM. The impact of diabetes mellitus and its co-morbidities on health care resources is profound; at present, $10 \%$ of total health care expenditure in UK is spent on the care of patients with diabetes (www.diabetes.org.uk, accessed 3 February 2012). The challenges in managing individual diabetes patients are sizeable and the future impact on health care resources is likely to exceed current predictions.

\section{Cardiovascular mortality and morbidity in T2DM}

Diabetes accounts for $6 \%$ of total global mortality, with half of the diabetes associated deaths attributed to cardiovascular disease (CVD) (www.idf.org, accessed 14 February 2012). Diabetes mellitus reduces life expectancy by a decade and it is generally accepted that $80 \%$ of patients with T2DM will develop and possibly die of macrovascular disease, predominantly resulting from cardiac causes. (http://diabetes.niddk.nih.gov/dm/pubs/ statistics, accessed 14 February 2012). Haffner and co-workers have shown that diabetic patients without a prior history of MI

GN Viswanathan, ${ }^{1}$ clinical research fellow; AG Zaman, ${ }^{1,2}$ consultant in cardiology

${ }^{1}$ Institute of Cellular Medicine, Newcastle University, UK; ${ }^{2}$ Freeman Hospital, Newcastle upon Tyne Hospitals NHS trust, Newcastle upon Tyne, UK carried the same risk of death as non diabetic patients with a previous MI. This has led to diabetes being called a cardiovascular disease equivalent. However, in the context of aggressive modern secondary prevention strategies; some authors have questioned this assumption. $^{2}$

In the presence of diabetes, the incidence of myocardial infarction (MI) is twice that in the general population and survival rates are lower than the norm. Although cardiovascular mortality has declined over the past decade, in patients both with and without diabetes, the relative risk remains unchanged with those with diabetes suffering a 2.4-fold increase in cardiovascular mortality, despite optimal evidence-based primary and secondary prevention strategies. ${ }^{3}$

Individuals with diabetes are twice as likely to be admitted to hospital. In a UK hospital admissions study, at any given time, nearly $10 \%$ of all in-patients had diabetes and experienced prolonged stays in hospital, resulting in about 80,000 additional bed days per year in the UK. In addition, those with diabetes account for 2.4 times greater medical expenditure per person when compared to a similar population without diabetes. ${ }^{4}$ Nevertheless, it is recognised that the most effective way of reducing the 'spiralling trend' of medical expenditure in patients with T2DM is to optimise strategies for preventing cardiovascular events. ${ }^{5}$

\section{Coronary artery disease in T2DM}

Atherothrombotic disease is responsible for significant mortality and morbidity in diabetes mellitus and associated with widespread changes in the coronary arteries, despite good control of diabetes mellitus and other cardiovascular risk factors. The pathophysiological mechanisms that contribute to coronary artery disease (CAD) in T2DM are multifactorial, complex and inter-linked. Contrary to earlier belief that CAD in diabetes is secondary to hyperglycaemia, several recent epidemiological studies and clinical follow-up studies have strongly suggested that factors other than hyperglycaemia play a significant role in the development of CAD in T2DM. ${ }^{6}$

Every stage in the process of coronary atherosclerosis initiation, progression and plaque rupture - is widespread and accelerated in T2DM. Coronary atherosclerotic burden in medium-sized arteries, along with microvascular disease, is consistently greater in T2DM. Besides, atherosclerotic changes from initiation to progression are more dynamic in T2DM, resulting in early plaque disruption and thus heralding more frequent occlusive atherothrombotic events. In addition, mild to moderate-sized atherosclerotic plaques, known as 'non-flow-limiting 
coronary atherosclerotic lesions' occur more frequently in $\mathrm{T} 2 \mathrm{DM}$ and are associated with the majority of the plaque rupture events and thromboses, resulting in non-ST-elevation acute coronary syndrome (NSTE-ACS) in T2DM. ${ }^{7}$

Excess atheroma burden in patients with T2DM is attributed to a loss of balance in the endothelial damage-repair cycle. In the presence of cardiovascular risk factors that are frequently associated with T2DM, such as hypertension, dyslipidaemia and obesity, damage to endothelium is more pronounced and repair mechanisms are rapidly exhausted, resulting in early and widespread atheromatous disease. ${ }^{8}$ In T2DM, atherosclerotic plaques are more prone to rupture (greater in number, with thinner fibrous caps, and increased numbers of inflammatory cells) and have been labelled as 'vulnerable plaque'. Plaque vulnerability can also be increased by other factors, such as increased shear stress of flowing blood in patients with T2DM. Greater levels of inflammatory mediators, such as cytokines and mononuclear phagocytes, can also resulting in a weakening of the protective fibrous cap. ${ }^{9}$

\section{Blood thrombogenicity in T2DM}

Thrombus formation is a protective mechanism by which haemostasis is maintained in a closed, high-pressure circulatory system after vascular damage. A balance exists between pathways that initiate thrombus formation and those that dissolve them. A highly specialised and efficient haemostatic system, consisting of cellular (platelets) and non-cellular (coagulation factors) components is responsible for the three main components of thrombosis, namely:

a) initiation of thrombosis,

b) propagation of thrombus,

c) auto-lysis of thrombus.

The enhanced thrombogenicity ('vulnerable blood') seen in T2DM is secondary to changes in all three components resulting from (amongst other causes):

a) hyperactive platelets,

b) higher levels of clotting factors, and

c) impaired fibrinolysis, which results in T2DM being classed as a 'prothrombotic state'.

The dysfunctional platelets of patients with T2DM respond to sub-threshold stimuli and are constantly active, even in the absence of a major plaque event; consequently, they have been labelled 'angry platelets.' ${ }^{10}$ Platelet-leucocyte interaction is responsible for the propagation and stabilisation of thrombi. T2DM is associated with greater platelet-leucocyte linkage mediated by P-selectin and cytokines. The release of procoagulant, oxidative and mitogenic stimulants thereby plays a significant role in stabilising arterial thrombi and capillary microembolisms (see Table 1).

Coagulation factor abnormalities are common in T2DM. In addition to conventional screening tests, such as shorter prothrombin and partial thromboplastin times (PT and APTT, respectively), T2DM is associated with elevated tissue factors, reduced tissue factor pathway inhibitor and higher levels of both prothrombin-activating factor and the thrombin-anti thrombin complex. Increased levels of factor VIII, plasma fibrinogen, Von Willebrand factor and factor XI found in T2DM also promote thrombus formation. In T2DM, reduced fibrinolysis potentiates thrombogenicity and also promotes atherogenesis by extending fibrin deposition. Plasminogen activator inhibitor-1 (PAI-1), the most potent inhibitor of the fibrinolytic system, is consistently elevated in the vessel walls of patients with T2DM. ${ }^{11}$

Inflammation is associated with arterial, venous and microvascular thrombosis; it has been widely accepted that inflammation

Table 1. Platelet alterations in T2DM.

- Increased aggregating responses

- Decreased responses to anti-aggregating substances

- Increased membrane viscosity

- Increased protein tyrosine phosphorylation

- Increased calpain activity

- Increased calcium content and reverse mode of sodium/calcium exchanger

- Increased calcium-induced presentation of the thrombospondin receptor CD36

- Reduced L-arginine uptake

- Reduced endothelial-type nitric oxide synthase activity

- Enhanced homocysteine ability to reduce nitric oxide availability

- Trend to leukocyte-platelet heterotypic aggregation

- Increased expression of adhesive proteins

- Decrease of nitric oxide availability

- Protein kinase $\mathrm{C}$ translocation to the membrane

Modified from Trovati and Anfossi. ${ }^{14}$

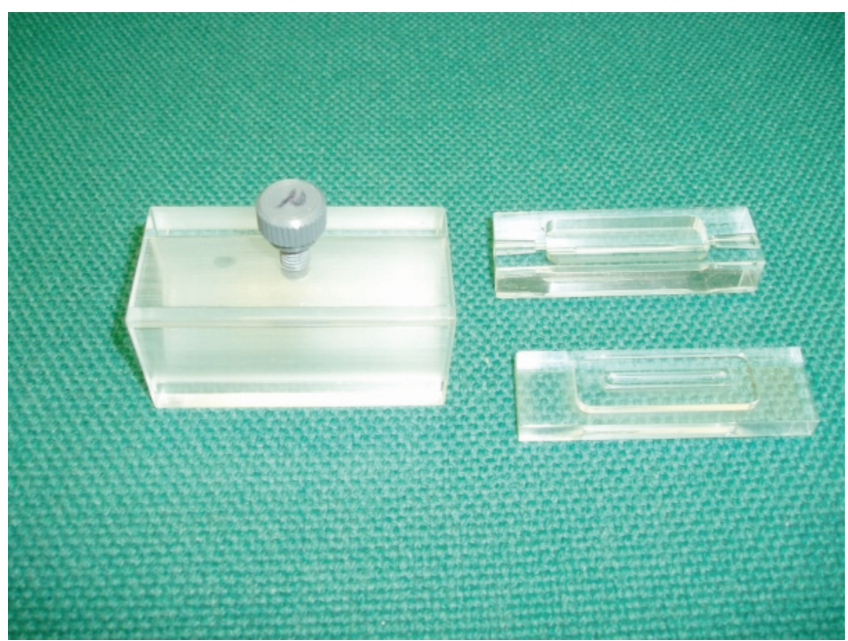

Fig 1. One of the high shear units of a Badimon perfusion chamber. There is a central groove between the two halves of the plexiglass chamber. Porcine aorta with its intima stripped off is placed in this unit as a thrombogenic substrate. This aorta is connected to blood flowing from the patients via a three-way tap. The thrombus laden aorta is fixed in buffered formalin and subsequently stained with modified Mason trichrome stain. 

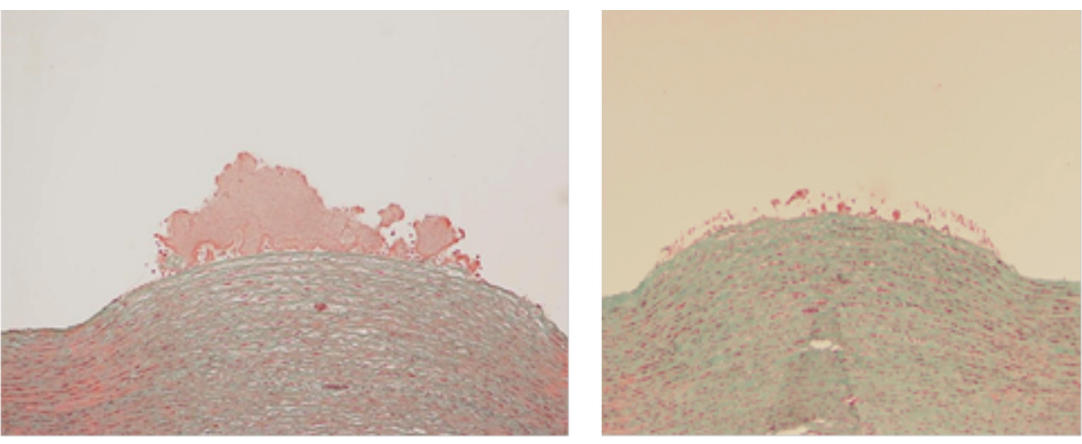

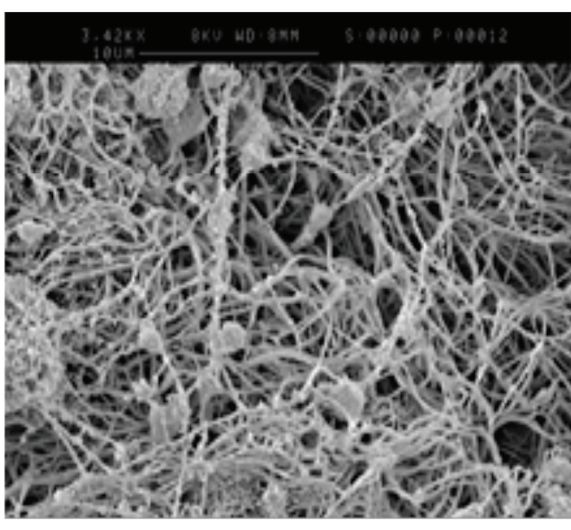

T2DM

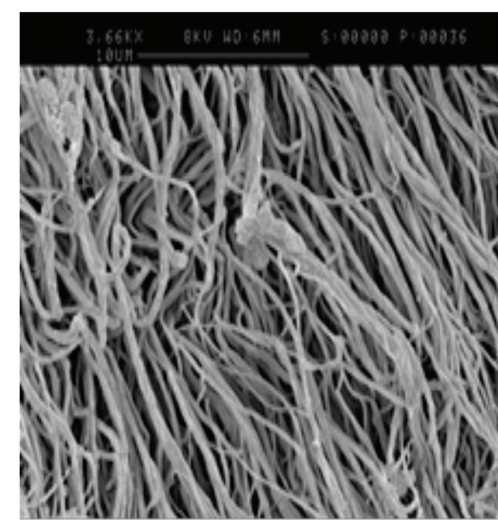

Non T2DM
Fig 2. Thrombus is present in greater quantity in arteries exposed to the blood of those with T2DM than in those exposed to blood from non-diabetic patients. The porcine arteries shown in the top panel were exposed to blood in a Badimon chamber and then stained with modified Mason trichrome stain. Scanning electron microscopy (bottom panel) showed thrombus formed from the blood of T2DM patients had more side-to-side linked fibrin with less twisted arrangement, accounting for its lower viscoelastic strength, when compared with thrombus formed from the blood of non-diabetic patients.

In a study of patients with diabetes, blood thrombogenicity was studied:

a) after NSTE-ACS (study 1), and

b) in stable CAD (study 2).

The hypothesis for study 1 (involving ACS patients) was that patients with type 2 diabetes mellitus have increased thrombus following NSTE-ACS, despite recommended secondary prevention therapy (www.clinicaltrials.gov, NCT00728286; www.ukcrn.org.uk, 7338).

The hypothesis for study 2 (of stable CAD patients) was that the addition of clopidogrel to standard aspirin treatment would be ineffective in reducing thrombus in patients with T2DM andCAD. (www.clinicaltrials.gov,NCT00728156; www.ukcrn.org.uk, 5159; Eudract number 2006003745-16; MHRA number 31088/0006/0010001).

The Badimon chamber (Fig 1), a validated $e x$ vivo arterial injury model, was used in both studies. This model simulates the in vivo situation of high-shear arterial wall damage and quantifies platelet-dependent thrombus, which is the endpoint of all in vitro haemostatic abnormalities. ${ }^{12}$

In patients presenting with NSTE-ACS, thrombus was significantly greater in T2DM than in those without diabetes, despite evidencebased optimal secondary prevention therapy. Upon examining the formed thrombus under electron microscopy, a marked difference in clot structure was seen (Fig 2). The fibrin strands

upregulates procoagulants and downregulates anticoagulants and fibrinolysis. Tumour necrosis factor alpha (TNF $\alpha)$ levels are elevated in T2DM patients and have been linked to the insulin resistance, thrombotic events and microvascular complications seen in T2DM. Suppression of TNF $\alpha$, by either biological antagonists or weight reduction and exercise, have been shown to improve glycaemic control in T2DM. ${ }^{11}$ It is plausible, but remains to be proven, that similar benefits in alleviating the prothrombotic state can be achieved in T2DM by pharmacological inhibition of cytokines.

\section{Contemporary studies of whole-blood thrombogenicity in patients with T2DM}

Recurrent cardiovascular events are more common in patients with T2DM, and recurrent sub-clinical thrombotic events might also accelerate atheroma progression. More detailed knowledge about blood thrombogenicity in T2DM can help clinicians to optimise antithrombotic therapy in this high-risk group. Better understanding of thrombogenicity in T2DM might help to identify novel therapeutic targets in the diabetic population. had an irregular pattern with crosslinking in the T2DM population (unpublished data, presented at the annual conference of the European Society for Cardiology, Munich, 2012). Using thromboelastography to assess the rate of thrombus lysis, a significantly slower rate of lysis was seen in patients with T2DM than in controls. Thrombus kinetics were found to be impaired when studied using thromboelastography. T2DM patients had lower visco-elastic tensile strength with a median clot index of -0.2 (interquartile range [IQR] -1.7 to 0.7 ) compared to 1.0 (IQR -0.9 to 3.3 ) in non-diabetic patients $(\mathrm{p}=0.04)$. The T2DM population also had impaired autolysis with a median rate of thrombus retraction $\mathrm{mm} / \mathrm{min}$ of 27.8 (IQR 11.7 to 70.7 ) compared to 78.8 in non-diabetic patients (IQR 68.5 to 109.6) $(\mathrm{p}<0.05)$. There was significant but moderate correlation between autolysis and thrombus quantity (Spearman's rho correlation coefficient $0.450, \mathrm{p}=0.02$ ) (unpublished data, presented at the Annual Professional Conference, Diabetes UK, 2010). The study revealed greater thrombus burden in T2DM patients, who had a mean thrombus area of $20,414 \mu \mathrm{m}^{2} / \mathrm{mm}$ (standard deviation [SD] $\pm 12,344 \mu \mathrm{m}^{2} / \mathrm{mm}$ ) compared to the mean of 14,933 $\mu \mathrm{m}^{2} / \mathrm{mm}(\mathrm{SD} \pm 8,415)(\mathrm{p}=0.023,95 \%$ confidence interval $811-$ $\left.10,674 \mu \mathrm{m}^{2} / \mathrm{mm}\right)$ in non-diabetic patients. ${ }^{13}$ 


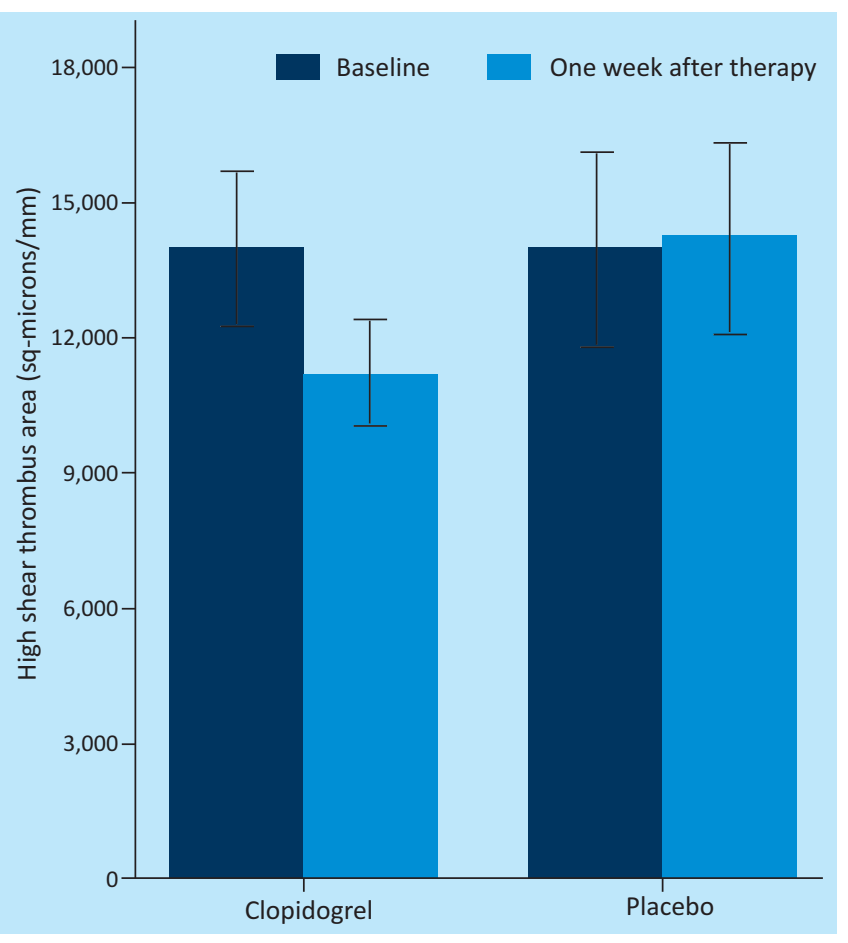

Fig 3. High-shear thrombus area measurements in patients at baseline and one week after treatment with either clopidogrel+aspirin or placebo+aspirin. In the clopidogrel group, thrombus area $\left(\mu \mathrm{m}^{2} / \mathrm{mm}\right.$, mean \pm SD) reduced from $13,978 \pm 5,501$ to $11,192 \pm 3,764$, whereas the change in the placebo group (from $13,959 \pm 7,038$ to $14,208 \pm$ $6780)$ was statistically insignificant $(p<0.0005)$. The mean difference in thrombus area resulting from clopidogrel treatment was 3,035 $\mu \mathrm{m}^{2} / \mathrm{mm}$ (95\% confidence interval 1,437-4,633 $\mu \mathrm{m}^{2} / \mathrm{mm}$ ). (Error bars: +/- 2 SE)

In patients with stable CAD, a randomised controlled study of the addition of clopidogrel or placebo to aspirin revealed a significant reduction in thrombus area (Fig 3). This suggests that the addition (to aspirin) of antiplatelet agents in patients with stable CAD and T2DM is effective in reducing thrombus. ${ }^{13}$

\section{Conclusions}

Patients who have T2DM and CAD suffer worse outcomes despite current aggressive prevention strategies. Blood thrombogenicity ('blood vulnerability') is higher in these individuals despite optimal antiplatelet therapy. Modifications of the vulnerable blood seen in these patients by addition of more or different anti-thrombotic agents, or by addition of medications that target different aspects of the coagulation pathway, might improve cardiovascular mortality. With the global pandemic of T2DM, coupled to the failure of aggressive risk-factor control to reduce macrovascular complications in the diabetic population, the need to study mechanisms that target thrombosis and reduce atherothrombotic complications is of paramount importance.

\section{References}

1 Wild S, Roglic G, Green A et al. Global prevalence of diabetes: estimates for the year 2000 and projections for 2030. Diabetes Care 2004;27:1047-53.

2 Bulugahapitiya U, Siyambalapitiya S, Sithole J, Idris I. Is diabetes a coronary risk equivalent? Systematic review and meta-analysis. Diabet Med 2009;26:142-8.

3 Preis SR, Hwang S-J, Coady S et al. Trends in all-cause and cardiovascular disease mortality among women and men with and without diabetes mellitus in the Framingham Heart Study, 1950 to 2005. Circulation 2009;119:1728-35.

4 Sampson MJ, Crowle T, Dhatariya K et al. Trends in bed occupancy for inpatients with diabetes before and after the introduction of a diabetes inpatient specialist nurse service. Diabetic Medicine 2006;23:1008-15.

5 Moore P. Type 2 diabetes is a major drain on resources. BMJ 2000;320:732.

6 Meier M, Hummel M. Cardiovascular disease and intensive glucose control in type 2 diabetes mellitus: moving practice toward evidencebased strategies. Vasc Health Risk Manag 2009;5:859-71.

7 Virmani R, Burke AP, Kolodgie F. Morphological characteristics of coronary atherosclerosis in diabetes mellitus. Can J Cardiol 2006;22 Suppl B:81B-84B.

8 Moreno PR, Fuster V. New aspects in the pathogenesis of diabetic atherothrombosis. J Am Coll Cardiol 2004;44:2293-300.

9 Hess K, Grant PJ. Inflammation and thrombosis in diabetes. Thromb Haemost 2011;105 Suppl 1:S43-54.

10 Bhatt DL. What makes platelets angry: diabetes, fibrinogen, obesity, and impaired response to antiplatelet therapy? J Am Coll Cardiol 2008;52:1060-1.

11 Grant PJ. Diabetes mellitus as a prothrombotic condition. J Int med 2007;262:157-72.

12 Natarajan A, Marshall SM, Worthley SG et al. The presence of coronary artery disease increases platelet-dependent thrombosis in patients with type 2 diabetes mellitus. J Thromb Haemost 2008;6:2210-3.

13 Viswanathan GN, Marshall SM, Schechter CB et al. Thrombus and antiplatelet therapy in type 2 diabetes mellitus. A prospective study after non-ST elevation acute coronary syndrome and a randomised, blinded, placebo-controlled study in stable angina. Thromb Haemost 2012 , in press.

14 Trovati M, Anfossi G. Mechanisms involved in platelet hyperactivation and platelet-endothelium interrelationships in diabetes mellitus. Curr Diab Rep 2002,2:316-22.

Address for correspondence: Dr AG Zaman,

Freeman Hospital, Newcastle upon Tyne NHS Hospital

Foundation Trust, Freeman Road, High Heaton,

Newcastle upon Tyne NE7 7DN.

Email: azfar.zaman@nuth.nhs.uk 